\title{
ERGONOMIA NO BAJA: ANÁLISE DO DESCONFORTO PERCEBIDO
}

\author{
Emilene Zitkus (1);
}

Ana Lya M. Ferrari (2);

Thais D. Reis (3);

Vanessa Usó (4);

Fausto O. Medola (5);

\section{Luis C. Paschoarelli (6)}

(1) UNESP 'Universidade Estadual Paulista', Doutora, Pesquisadora Pós-Doc

e-mail: emilenezitkus@gmail.com

(2) UNESP 'Universidade Estadual Paulista', Graduanda, Bolsista Iniciação Científica email: ana.lya.mf@gmail.com

(3) UNESP 'Universidade Estadual Paulista', Graduanda, Bolsista Projeto de Extensão e-mail:tdeloroso@gmail.com

(4) UNESP 'Universidade Estadual Paulista', Graduanda e-mail: vanessauso@hotmail.com

(5) UNESP 'Universidade Estadual Paulista', Professor Doutor e-mail: fausto.medola@faac.unesp.br

(6) UNESP 'Universidade Estadual Paulista', Professor Doutor e-mail: paschoarelli@faac.unesp.br

\begin{abstract}
RESUMO
O presente estudo é parte de um projeto integrando ergonomia ao projeto Baja SAE. Um dos objetivos foi analisar o desconforto percebido pelos pilotos ao conduzir dois veículos, o Baja Antigo (BA) - modelo 2013 - e o Baja Novo (BN) - modelo 2015. Em média os pilotos sentiram maior desconforto após conduzirem o BN, sendo as regiões das costas as que sofreram maior impacto. As repostas dadas pelos pilotos foram contrastadas com os ângulos posturais, sendo aqueles que mais diferem dos ângulos de conforto sugeridos pela literatura, os correspondentes às regiões corporais com maior incômodo ou dor.
\end{abstract}

\section{ABSTRACT}

The present study is part of an ergonomics research project involving the Baja SAE project. One of the objectives of the study was to analyse the body areas of discomfort among pilots of the old Baja (BA) used in competitions in 2015 - and the new Baja (BN) - used in 2016. On average, the pilots felt the $B N$ more uncomfortable, and the back was the body part with major discomfort or pain. The answers given by the pilots were cross-compared with the postural angles measured within the sample. The angles that greatly differ from those recommended in the literature, were related to the painful body 
areas.

\section{INTRODUÇÃO}

A Sociedade de Engenheiros Automotivos (Society of Automotive Engineers - SAE) criou na década de 1970 a competição dos veículos Baja SAE entre estudantes de engenharia. A competição envolve o projeto, desenvolvimento e produção dos veículos, vislumbrando os elementos de projetos de engenharia da indústria automotiva. Como na indústria, as equipes são divididas em times responsáveis por differentes partes que compõe o veículo, como powertrain, chassi, entre outros. Numa iniciativa multidisciplinar a equipe do Baja - UNESP Bauru, buscou integrar ergonomia na avaliação de dois modelos atuais, o Baja Antigo (BA) modelo 2013 - e o Baja Novo (BN) - modelo 2015 (Figura 1).

Figura 1 - Modelos: Baja Antigo (BA) à esquerda e Baja Novo (BN) à direita, usados nos testes

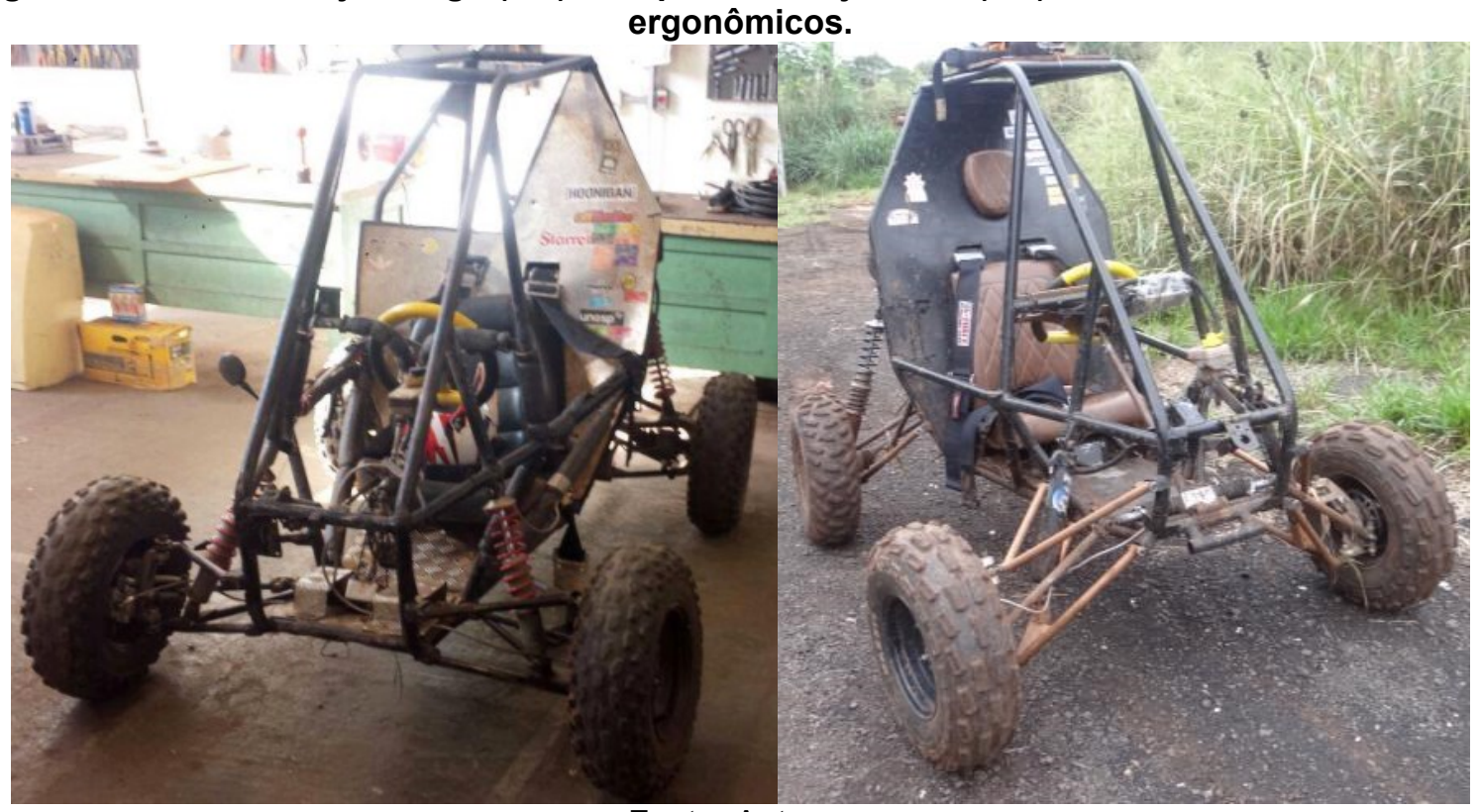

Fonte: Autores

Dentre os requisitos de design do Baja, são determinados pela SAE os seguintes aspectos:

- Possibilite o transporte de uma pessoa de $1900 \mathrm{~mm}$ de altura, pesando até $113,4 \mathrm{~kg}$.

- Largura máxima de 1620 mm e comprimento irrestrito.

- Ter capacidade de ser conduzido com segurança sobre terreno com obstáculos.

- O tamanho interno da gaiola (cockpit) deve possuir espaço suficiente para: 1) o capacete do condutor estar $152,4 \mathrm{~mm}$ de distancia de quaisquer pontos na cabine do veiculo, exceto o banco do piloto e os suportes traseiros de segurança. 2) possuir espaço livre de $76,2 \mathrm{~mm}$ entre o revestimento da estrutura entre 0 tórax do condutor, joelhos, ombros, cotovelos, mãos e braços.

Uma das avaliações ergonômicas utilizadas nesse projeto foi a análise do desconforto percebido pelos pilotos ao testarem os dois modelos Bajas. Tal análise será apresentada e discutida no presente artigo.

O conforto do piloto de um veículo está diretamente relacionado com pelo menos três fatores: sua postura, a vibração do veículo e o tempo realizando a tarefa de condução. A postura sentada por si só pode ser fonte de desconforto após certo período ininterrupto (HUET e MORAES, 2003). Nessa postura, a pressão nas vértebras lombares é alta, a posição da coluna é alterada, posicionando-se reta ou com curvatura acentuada para frente 
na sua parte superior (cifose) (HOFFMANN e JUNIOR, 2010). Essa posição ainda causa o aumento de pressão no osso ísquio das nádegas, e exige atividade muscular do dorso e do ventre para mantê-la. Dessa forma, o consumo de energia é de 3 á $10 \%$ maior em relação a posição horizontal. Por este motivo, lida (2005) indica que, em veículos de passeio, o assento deve possibilitar mudanças de postura, a fim de evitar a fadiga.

"Para que o ambiente interno de um veículo seja considerado confortável dependerá dos espaços livres entre o condutor e os limites de alcance aos diversos comandos. Ao dirigir um automóvel, o motorista mantém uma postura sentada e estática, caracterizada por exigir contração contínua de alguns músculos na região do dorso e do ventre." (SANTOS, 2015).

O desconforto causado por longos períodos de condução aliados às condições posturais são algumas das principais preocupações quando se trata do estudo da ergonomia em automóveis. Nesses casos, os pés não servem apenas de apoio, mas também devem ser utilizados para ativar os pedais de aceleração, freio e embreagem. Segundo Fai et al. (2007) o contato do condutor com o assento é uma significativa fonte de desconforto, tendo como causas principais a rigidez e o design do assento e os materiais utilizados em sua confecção.

O banco de um veículo, especialmente o motorista, deve, além de permitir regulagens a fim de se adequar a cada condutor, se atentar com relação aos materiais utilizados em sua confecção. Materiais que permitam a transpiração, não sejam escorregadios e cuja densidade seja adequada para absorver os impactos e vibrações são os mais indicados. Zenk (2012), afirma em seu estudo que quanto melhor a distribuição do peso no assento, menor será a pressão entre os discos intervertebrais da lombar. Assim, o conforto do assento não deve se restringir a favorecer uma postura confortável, mas também facilitar o acesso aos pedais, volantes e demais controles do veículo, de forma que todos eles estejam ao alcance do condutor sem esforços extras dos braços e da coluna.

O chamado $H$-Point é o ponto da articulação do quadril, utilizado como base para avaliação de dimensões ou ângulos posturais dos motoristas, ou seja, o ponto de partida para a análise ergonômica de veículos (Figura 2). Uma série de estudos avaliou o conforto postural a partir de amostras de pessoas de diferentes nacionalidades, recomendando diversos intervalos de ângulos para os membros superiores, inferiores, tronco - coxas e troncobraços. A figura 3 mostra a localização de quatro dos ângulos posturais recorrentes em estudos de análise postural de veículos automotivos, e que foram também avaliados no presente estudo.

Figura 2 - Hpoint e suas variações entre motoristas de menor e maior estatura.

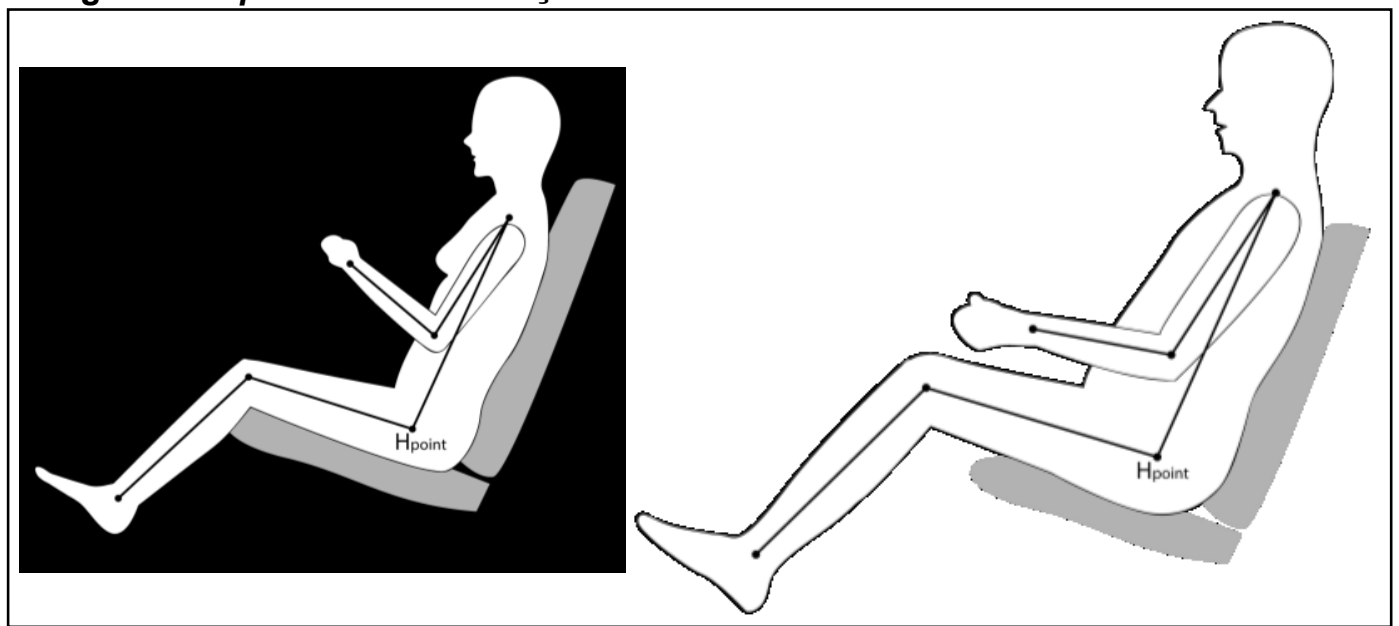

Fonte: Autores 
Figura 3 - Ângulos analisados no estudo: A (ângulo ombro), B (ângulo cotovelo), C (troncocoxa), D (joelho).

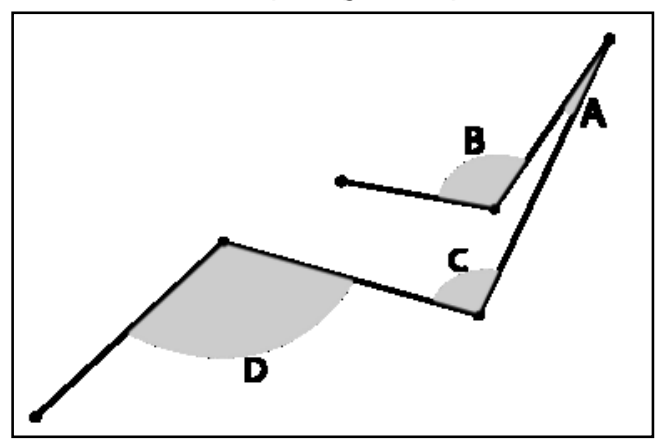

Fonte: Autores

Os intervalos de ângulos apresentados na tabela abaixo são baseados em estudos que mediram ângulos de conforto entre participantes de diferentes estaturas, em alguns casos com estaturas representando o percentil $5^{\circ}$ e $95^{\circ}$, em outros representando do percentil $1^{\circ}$ ao $99^{\circ}$ e outros não relacionados ao percentil da população. A maior variação se dá entre estudos analisando populações de origem caucasiana (REBIFFE, 1969; GRANDJEAN, 1980; TILLY e DREYFUSS, 1993; PORTER e GYI, 1998; HANSON et al., 2005), população coreana (PARK et al., 2000) e população da Malásia (MOHAMAD et al., 2010), exatamente pelas diferenças antropométricas das diferentes origens.

Tabela 1 - Intervalos de ângulos A, B, C e D em diversos estudos.

\begin{tabular}{|c|c|c|c|c|}
\cline { 2 - 5 } \multicolumn{1}{c|}{} & \multicolumn{4}{|c|}{ Intervalo do mínimo ao máximo ângulo de conforto } \\
\cline { 2 - 5 } & $\mathrm{A}$ & $\mathrm{B}$ & $\mathrm{C}$ & $\mathrm{D}$ \\
\hline Rebiffe (1969) & $10-45$ & $80-120$ & $95-120$ & $95-135$ \\
\hline Grandjean (1980) & - & - & $100-120$ & $110-130$ \\
\hline Tilly \& Dreyfuss (1993) & $0-35$ & $80-165$ & $95-100$ & $110-120$ \\
\hline Porter \& Gyi (1998) & $19-75$ & $86-164$ & $90-115$ & $99-138$ \\
\hline Park et al (2000) & $7-37$ & $86-144$ & $103-131$ & $120-152$ \\
\hline Hanson et al (2005) & $14-68$ & $96-160$ & $92-109$ & $109-157$ \\
\hline Mohamad et al (2010) & - & $100-167$ & $96-123$ & $102-143$ \\
\hline
\end{tabular}

Fonte: Autores

Em sua revisão bibliográfica, Hoffmann e Junior (2010) analisaram diversos artigos dos quais extrairam recomendações para melhorar as questões posturais nos assentos de veículos:

- Formato do assento deve acompanhar a curvatura da coluna, sem retifica-la, uma vez que apoios na coluna dorsal diminuem a pressão nos discos intervertebrais.

- A dimensão ântero-posterior do assento deve apoiar totalmente as coxas, entretanto, não deve comprimir a região posterior dos joelhos.

- Apoio lombar não deve ser elevado a fim de não comprometer a movimentação dos membros superiores.

Além da postura adotada pelo motorista ou piloto, existem outros fatores que afetam a sensação de conforto. Para avaliar o conforto de diferentes assentos de automóveis, Kolich (2003) comparou a adequação dimensional recomendada em estudos antropométricos e posturais, com o conforto percebido pelos participantes de sua pesquisa. Apesar de alguns assentos terem as mesmas medidas e adequações antropométricas a percepção de 
conforto variou. Por exemplo, as dimensões (altura e largura) do contorno lombar de dois assentos eram idênticas, mas não foram percebidas com o mesmo nível de conforto, sugerindo que a região lombar possa ser afetada pelo conforto da coxa. O autor sugere que conforto de assento seja estudado extensivamente relacionando outras variáveis, além de antropometria e ângulos posturais (KOLICH et al., 2004).

Com o objetivo de entender o desconforto percebido pelos pilotos ao conduzir dois veículos Baja, o presente estudo buscou analisar dados subjetivos de desconforto, combinados com dados posturais. Desta maneira, segue-se os métodos empregados.

\section{MATERIAIS E MÉTODOS}

A coleta de dados foi realizada em áreas adjacentes ao Campus da UNESP de Bauru. O trajeto de 1,6 km foi escolhido por apresentar características semelhantes às das pistas offroad para as quais os veículos Baja SAE são projetados. A coleta foi conduzida conforme o Código de Deontologia do Ergonomista Certificado, Norma ERGBR 1002 (ABERGO, 2003).

\subsection{Participantes}

Participaram do estudo 14 voluntários, todos do gênero masculino e membros da Equipe Pac Baja da Faculdade de Engenharia da UNESP de Bauru, com experiência na condução dos dois veículos avaliados - o BA e o BN. Para a avaliação de análise postural, foram analisados quatro dos participantes (veja tabela 2), o membro mais baixo $(1,67 \mathrm{~m})$ e o mais alto $(1,90 \mathrm{~m})$; bem como o maior Índice de Massa Corporal - IMC $(34,7)$ e o menor IMC (19.3).

Tabela 2 - Participantes e suas respectivas altura, peso e IMC.

\begin{tabular}{|c|c|c|c|}
\hline & ALTURA & PESO & IMC \\
\hline P1 & $1,90 \mathrm{~m}$ & $74 \mathrm{~kg}$ & 20.5 \\
\hline P2 & $1,75 \mathrm{~m}$ & $60 \mathrm{~kg}$ & 19.6 \\
\hline P3 & $1,67 \mathrm{~m}$ & $67 \mathrm{~kg}$ & 24.0 \\
\hline P4 & $1,76 \mathrm{~m}$ & $67,5 \mathrm{~kg}$ & 21.8 \\
\hline P5 & $1,86 \mathrm{~m}$ & $120 \mathrm{~kg}$ & 34.7 \\
\hline P6 & $1,79 \mathrm{~m}$ & $62 \mathrm{~kg}$ & 19.3 \\
\hline P7 & $1,80 \mathrm{~m}$ & $102 \mathrm{~kg}$ & 31.5 \\
\hline P8 & $1,90 \mathrm{~m}$ & $93 \mathrm{~kg}$ & 25.7 \\
\hline P9 & $1,74 \mathrm{~m}$ & $68 \mathrm{~kg}$ & 22.4 \\
\hline P10 & $1,85 \mathrm{~m}$ & $69,5 \mathrm{~kg}$ & 20.3 \\
\hline P11 & $1,83 \mathrm{~m}$ & $104 \mathrm{~kg}$ & 31.0 \\
\hline P12 & $1,90 \mathrm{~m}$ & $110 \mathrm{~kg}$ & 30.5 \\
\hline P13 & $1,82 \mathrm{~m}$ & $92,5 \mathrm{~kg}$ & 27.9 \\
\hline P14 & $1,71 \mathrm{~m}$ & $80,5 \mathrm{~kg}$ & 27.5 \\
\hline
\end{tabular}

Fonte: Autores

\section{$2.2 \quad$ Materiais}

Um Termo de Consentimento Livre e Esclarecido (TCLE) foi elaborado, assim como um questionário de identificação com dados pessoais de cada voluntário. Para a avaliação de desconforto foi utilizado um questionário elaborado pelos autores, adaptando os diagramas de Corlett e Manenica (1980) e os do Nordic Muscular Skeletal Questionnaire (Kuorinka et al., 1987 apud: Phanthanosy, et al., 2011). A Figura 4 apresenta o diagrama corporal (Frente e Costas) dividido em 18 partes, cada uma delas com uma escala correspondente com valores de 1 a 5 , onde 1 equivale a nenhum desconforto e 5 a extremamente desconfortável, conforme figura 4. Também foi utilizado no estudo o software gratuito SAPO - Software para Avaliação Postural (SOUZA et al., 2011) para medir as angulações posturais a partir de registro fotográfico. 
Figura 4 - Modelo usado para o questionário de desconforto percebido.

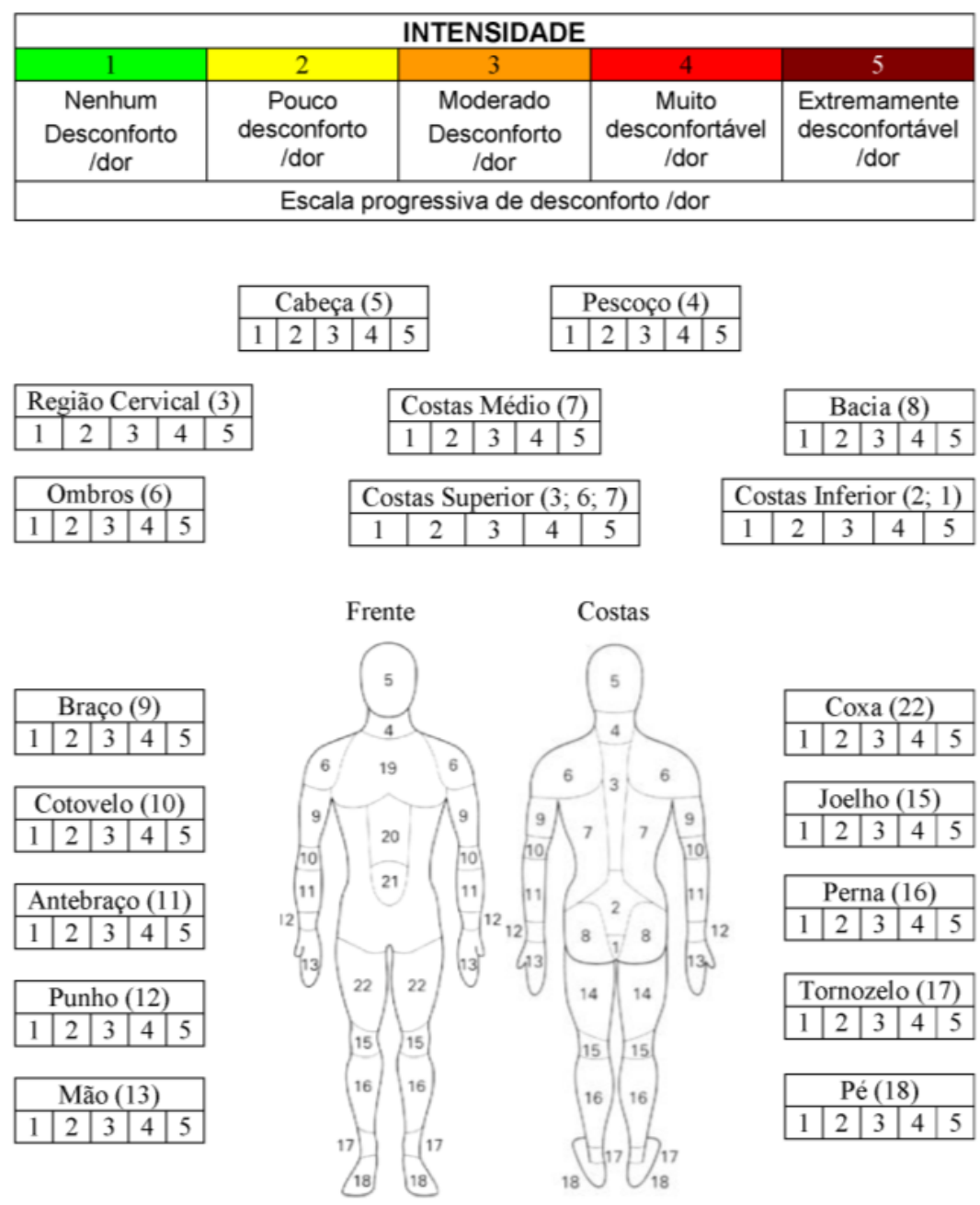

Fonte: Autores

\subsection{Procedimento}

No estudo de percepção de desconforto, cada voluntário recebeu e preencheu o TCLE junto com o questionário de identificação, seguido do questionário de desconforto antes de pilotar cada um dos carros. Estes questionários anteriores á atividade tinham como objetivo verificar qualquer desconforto pré-existente à condução do veículo. Após o preenchimento do questionário anterior à atividade, o voluntário realizava o percurso previamente definido com um dos carros, e em seguida respondia o questionário posterior à atividade, no qual relatava o desconforto percebido. Após aproximadamente 15 min de repouso, o processo era repetido, com a aplicação do questionário anterior à atividade, a corrida no trajeto com o segundo carro e a aplicação de um novo questionário posterior à atividade referente ao segundo carro pilotado. A ordem de utilização dos carros foi aleatorizada para cada voluntário.

A avaliação postural foi realizada através de análises por fotogrametria. Para tanto, cada um dos quatro participantes dessa etapa do estudo tiveram marcadores de superfície autoadesivos colocados nos pontos anatômicos indicados pelo tutorial do software SAPO. Foram 6 pontos marcados: Três nos membros superiores (acrômio, epicôndilo latera e o ponto médio entre o processo estilóide do rádio e a cabeça da ulna) e três nos membros inferiores (trocânter maior do fêmur, linha articular do joelho e maléolo lateral). Além disso, seguindo as recomendações para a calibragem da imagem no software, foi utilizado um fio de prumo com dois pesos presos a uma distancia de 1 metro um do outro, pendurado 
verticalmente ao lado de cada carro. Isso possibilitou que a imagem fosse ajustada a partir dessa linha vertical $\left(90^{\circ}\right.$ pré-estabelecida). Os quatro voluntários foram fotografados em vista lateral do perfil esquerdo, acomodados no interior do cockpit de cada um dos carros simulando a posição adotada para condução. As angulações posturais foram calculadas através do software SAPO e foram comparadas com as angulações posturais já recomendadas pela literatura. É importante ressaltar que as medidas propostas na literatura, geralmente consideram indivíduos que correspondam aos 5\% il e 95\% il, entretanto, nesse estudo não foram avaliados sujeitos de medidas relativas a esses percentis, e sim entre o maior e o menor participante do estudo.

\section{RESULTADOS E DISCUSSÃO}

Em média os participantes sentiram maior desconforto após pilotarem o BN. A Figura 5 apresenta a média do desconforto percebido em cada uma das regiões corporais, sendo a região lombar da coluna - médio, inferior e cervical - aquelas percebidas como as mais desconfortáveis. A Figura 6 apresenta, através dos três gráficos apresentados, a média dos 14 participantes com relação ao desconforto de cada região corporal, sendo possível notar que apesar de muitas regiões terem sido identificadas como verde e amarelo na Figura 5, algumas das regiões estão no limiar do pouco desconforto, como o antebraço para o BA; e a coxa e o joelho para o BN. De forma geral, em média, o BA ainda se apresenta mais confortável para os pilotos participantes do estudo.

Figura 5 - Média de desconforto percebido pelos pilotos do Baja Antigo (BA) e Baja Novo (BN).

\begin{tabular}{|c|c|c|c|c|}
\hline \multicolumn{5}{|c|}{ INTENSIDADE } \\
\hline 1 & 2 & 3 & 4 & 5 \\
\hline $\begin{array}{c}\text { Nenhum } \\
\begin{array}{c}\text { Desconforto } \\
\text { /dor }\end{array}\end{array}$ & $\begin{array}{c}\text { Pouco } \\
\text { desconforto } \\
\text { /dor }\end{array}$ & $\begin{array}{c}\text { Moderado } \\
\text { Desconforto } \\
\text { /dor }\end{array}$ & $\begin{array}{c}\text { Muito } \\
\text { desconfortável } \\
\text { /dor }\end{array}$ & $\begin{array}{c}\text { Extremamente } \\
\text { desconfortável } \\
\text { /dor }\end{array}$ \\
\hline
\end{tabular}

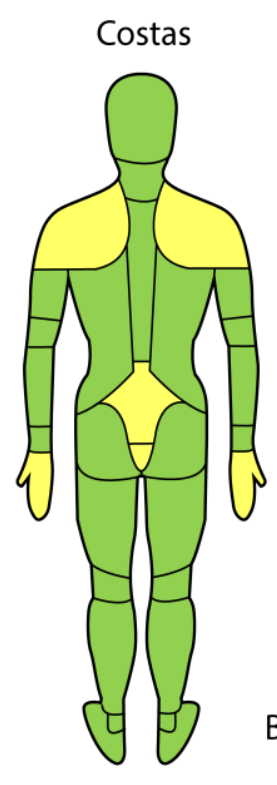

Baja Antigo
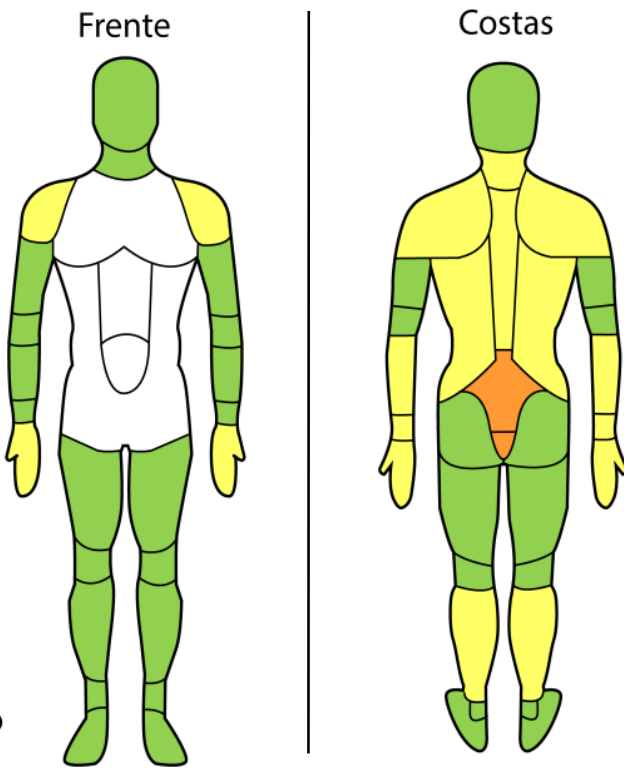

Baja Novo

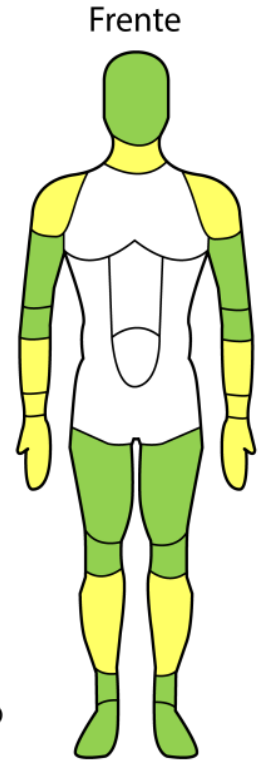

Fonte: Autores 
Figura 6 - Gráficos com as médias de desconforto percebido em diferentes regiões corporais.
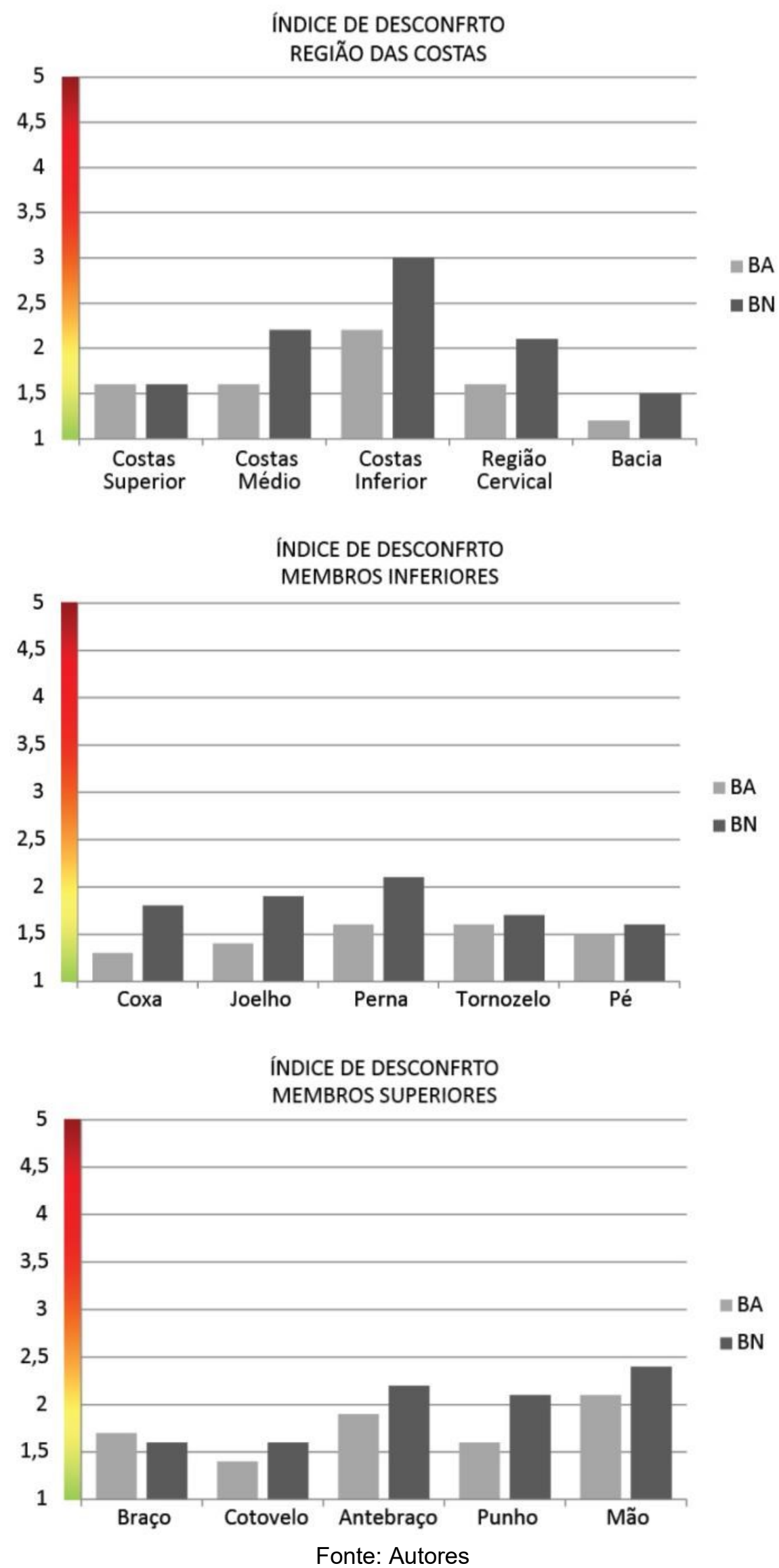
A diferença entre as respostas para o desconforto percebido na região do tronco foi bastante discrepante entre os dois modelos de veículos analisados, principalmente nas costas inferior - região Lombar. Enquanto após usar o BA, seis dos 14 pilotos não sentiram desconforto, dois sentiram pouco desconforto e seis desconforto moderado; no caso do BN, apenas dois não sentiram desconforto e dois referiram pouco desconforto, mas 10 dos 14 participantes sentiram desconforto acima do moderado: seis com desconforto moderado, três classificando como muito desconfortável e um com desconforto extremo.

Para melhor entender as causas do desconforto, foram analisados os dados posturais a partir da fotogrametria de quatro pilotos - especificados na seção 2.1. Figura 7 apresenta os de maior e menor estatura.

Figura 7 - Análise postural através de fotogrametria: acima piloto com menor estatura e abaixo o com maior

\section{Piloto com menor estatura}

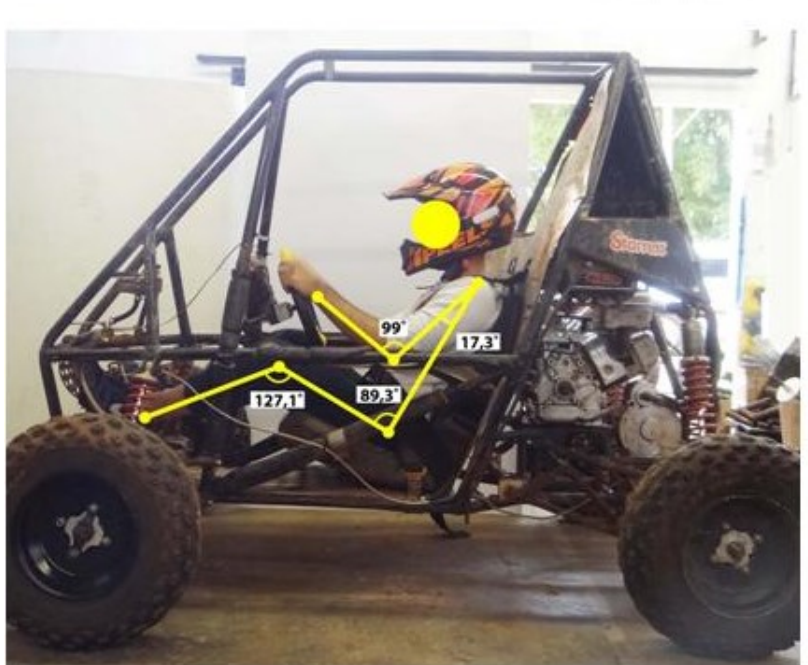

Baja Antigo

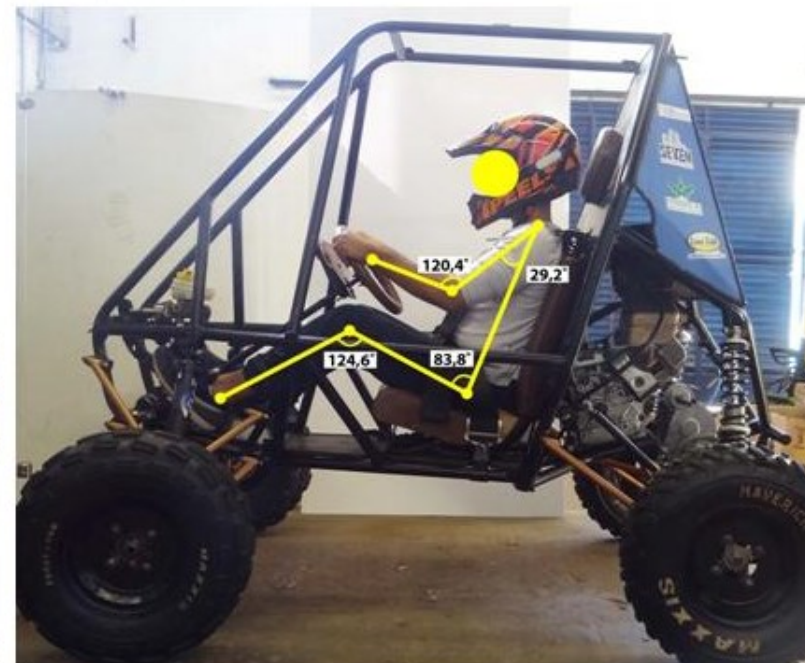

Baja Novo

Piloto com maior estatura

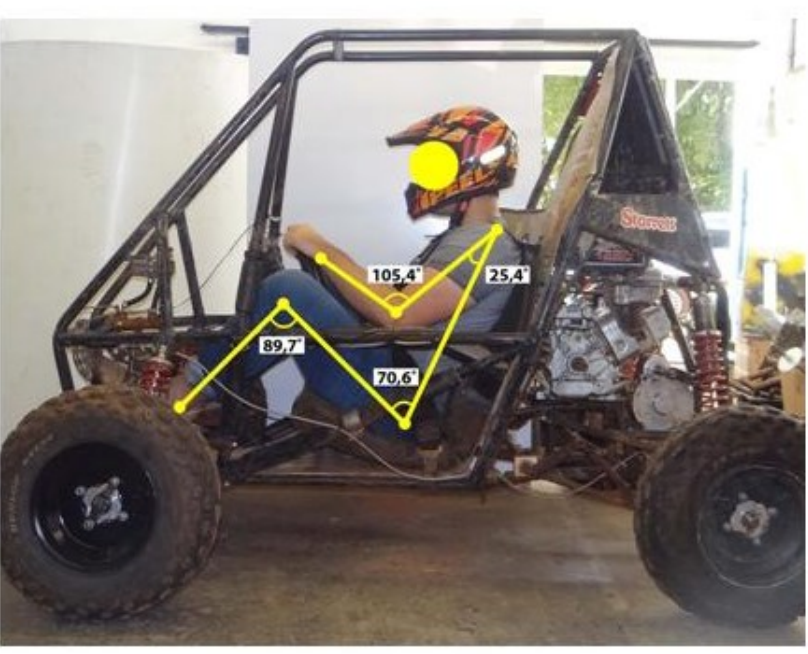

Baja Antigo

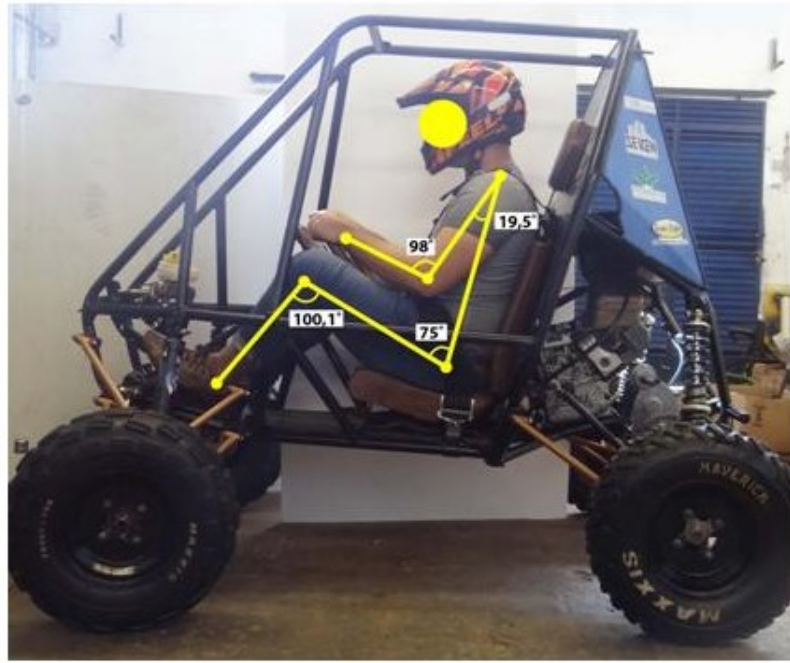

Baja Novo

Fonte: Autores

A Tabela 3 destaca os valores dos ângulos de ambos os pilotos. Comparando com a tabela apresentada anteriormente (Tabela 1) e o desconforto percebido nos pilotos, pode-se notar que os ângulos que mais se aproximam dos valores recomendados estão relacionados a 
menor intensidade de desconforto/dor no membro que o ângulo se refere. A (ângulo ombro), B (ângulo cotovelo), C (tronco-coxa), D (joelho).

Tabela 3 - Intervalos de ângulos A, B, C e D nos pilotos de menor e maior estatura.

\begin{tabular}{|c|c|c|c|c|c|}
\cline { 3 - 6 } \multicolumn{2}{c|}{} & \multicolumn{4}{|c|}{ Ângulos observados nos pilotos de menor e maior estatura } \\
\cline { 3 - 6 } \multicolumn{2}{c|}{} & A & B & C & D \\
\hline \multirow{3}{*}{ Menor } & B.A. & 17,3 & 99 & 89,3 & 127,1 \\
\cline { 2 - 6 } & B.N. & 29,2 & 120,4 & 83,8 & 124,6 \\
\hline \multirow{3}{*}{ Maior } & B.A. & 25,4 & 105,4 & 70,6 & 89,7 \\
\cline { 2 - 6 } & B.N. & 19,5 & 98 & 75 & 100,1 \\
\hline
\end{tabular}

Fonte: Autores

Apesar dos ângulos relacionados aos membros superiores (A e B) estarem dentro dos intervalos recomendados (HANSON, 2006; PARK et al., 2000, PORTER e GYI, 1998; TILLY e DREIFUSS, 1993 e REBIFFE, 1969) em ambos os modelos, eles não refletem as mesmas percepções de desconforto nos BA e no BN de acordo com o gráfico dos membros superiores apresentado na Figura 6 . Já o desconforto nas costas, principalmente para os pilotos mais altos pode ser reflexo de que o mínimo de $95^{\circ}$ recomendado para o ângulo ' $\mathrm{C}$ ' (tronco-coxa) não foi atendido. No entanto, outros fatores afetam a percepção de desconforto, tais como a posição estática e a necessidade de manejar os controles (IIDA, 2005); a forma do assento e o material utilizado em sua confecção (FAl et al., 2007); a distribuição da pressão (ZENK, 2012); e o reflexo do desconforto de um (ou vários) membro(s) que afetam outras regiões corporais (KOLICH, 2003). Dessa maneira, além do ângulo postural, outros fatores possivelmente contribuíram para a sensação de desconforto nos pilotos, principalmente no Baja Novo.

\section{CONCLUSÃO}

O presente estudo encontrou que diferenças no design do veículo influenciam a posição do piloto e, desta forma, a percepção de desconforto, nesse caso particularmente, a região Lombar da coluna foi bastante afetada pelas variações do design. De fato, a sucinta revisão bibliográfica sobre alguns dos fatores que afetam a percepção de conforto, combinada com os resultados do presente estudo indicam que a adequação dos pilotos aos intervalos de ângulos de conforto por si só não melhoram o conforto percebido. É necessário combinar diversos fatores, como formato do assento para melhor distribuição do peso; espessura e materiais do assento e encosto do banco; o espaço do cockpit e a adequação dos controles (inclinação e dimenção do painel, do volante, etc) para que todas as regiões do corpo estejam em boa postura e conforto. Assim sendo, estudos mais abrangentes sobre esses fatores podem contribuir para designs de novos veículos mais adequados ergonomicamente.

\section{AGRADECIMENTOS}

Este estudo obteve apoio da CAPES - Coordenação de Aperfeiçoamento de Pessoal de Nível Superior. 


\section{REFERÊNCIAS BIBLIOGRÁFICAS}

ASSOCIAÇÃO BRASILEIRA DE ERGONOMIA. ERG BR 1002: Código de Deontologia do Ergonomista Certificado. Ouro Preto: Minas Gerais, 2003.

BARBOSA, G.S. Análise de sistema manual de controle de caixa de transmissão veicular - uma abordagem ergonômica. 2007. 106 f. Dissertação (Mestrado) - Escola Politécnica da Universidade de São Paulo. São Paulo.

BERGMANA, C., et al. Implementation of suitable comfort model for posture and motion prediction in DHM supported vehicle design. 6th International Conference on Applied Human Factors and Ergonomics (AHFE 2015). Procedia Manufacturing. Vol. 3, p. 3753 - 3758. 2015.

CORLETT, E.N.; MANENICA, I. The effects and measurement of working postures. Applied Ergonomics, Trondheim, v.11, n.1, p. 7-16, march. 1980.

DREYFUSS, $H$. As medidas do homem e da mulher: Fatores humanos em design. Porto Alegre: Bookman. 2005. Apud: SILVA, et al. A Ergonomia na Identificação dos aspectos dimensionais críticos: o estudo antropométrico de um carro de competição BAJA SAE. Human Factors in Design, v.2, n.3, p.1-19. 2013. p.8.

FAI, T.C.; DELBRESSINE, F.; RAUTERBERG, M. Vehicle Seat Design: State of the Art and Recent Development. Proceedings World Engineering Congress 2007. Penang, Malaysia. p. 51-61. 2007.

GRANDJEAN, E. Sitting posture of car drivers form the point of view of ergonomics. Human Factors in Transport Research. Vol. 2, p. 205-213. 1980. Apud: MOHAMAD, D. et al. Integration of Comfort into a Driver's Car Seat Design Using Image Analysis. American Journal of Applied Sciences. Vol. 7, n. 7. p. 937-942. 2010. p. 938.

HANSON, L. A; SPERLING, L.; AKSELSSON, R. Preferred car driving posture using 3-D information. International Journal of Vehicle Design. Vol. 42, n. 1-2. p. 154-169. 2005.

HOFFMANN, A.R.; JúNIOR, W,H. A Análise de Postos de Condução de Veículos de Passeio Para Uso Profissional Sob a Ótica da Ergonomia: Estudo de caso realizado com motoristas de táxi em São Paulo. Revista - E-FAPPES, São Paulo, vol. 01, nº 01, Jan-Jun 2010.

HUET, M; MORAES, A. Medidas de Pressão sob a Pelve na Postura Sentada em Pesquisas de Ergonomia. Fisioterapia Brasil. Vol 4. N. 6. Nov/Dez. 2003.

IIDA, lida. Ergonomia: Projeto e Produção. 2. ed. São Paulo: Edgard Blücher, 2005.

KUORINKA, I et al. Standardized Nordic questionnaires for the analysis of musculoskeletal symptoms. Applied Ergonomics. Elsevier Science. Vol.18, p. 233-237. 1987. Apud: PHANTHANOSY, N. et al. Baja SAE All Terrain Vehicle. Presentation given at Loughborough University in 11 December 2011. Disponível em: <http://www.slideshare.net/ paulodavila/sae-baja-ergonomic-evaluation> Acesso em: 02 jun 2016.

$\mathrm{KOLICH}, \mathrm{M}$, et al. Automobile seat comfort prediction: statistical model vs. artificial neural network. Applied Ergonomics. Elsevier Science. Vol. 35, p. 275 - 284. 2004.

$\mathrm{KOLICH}, \mathrm{M}$. Automobile seat comfort: occupant preferences vs. anthropometric accommodation. Applied Ergonomics. Elsevier Science. Vol. 34, p. 177-184. 2003.

MOHAMAD, D. et al. Integration of Comfort into a Driver's Car Seat Design Using Image Analysis. American Journal of Applied Sciences. Vol. 7, n. 7. p. 937-942. 2010.

PARK, S.J., et al. Comfortable driving postures for Koreans. International Journal of Industrial Ergonomics. Vol. 26, n. 4, p. 489-497. 2000. 
PORTER, J.M.; GYI, D.E. Exploring the optimum posture for driver comfort. International Journal of Vehicle Design. Inderscience. Vol. 19, n 3., p. 255- 266. 1998.

REBIFFE, R. An ergonomic study of arrangement of the driving positions in motorcars, Proceedings of Symposium on Sitting Posture, Zurich, Switzerland, p.132-147. 1969. Apud: BERGMANA, C., et al. Implementation of suitable comfort model for posture and motion prediction in DHM supported vehicle design. 6th International Conference on Applied Human Factors and Ergonomics (AHFE 2015). Procedia Manufacturing. Vol. 3, p. 3753 3758. 2015. p. 3755.

SANTOS, A.B. Estudo ergonômico do veículo BAJA do centro universitário UNIVATES. 2015. Monografia (Graduação em Engenharia de Produção) - Centro Universitário UNIVATES, Lajeado, 2015.

SAPO. Tutorial para localização dos pontos anatômicos. Disponível em: <http://docslide.com.br/documents/tutorial-sapo-pontos-anatomicos.html> Acesso em: 08 jul 2016.

SILVA, et al. A Ergonomia na Identificação dos aspectos dimensionais críticos: o estudo antropométrico de um carro de competição BAJA SAE. Human Factors in Design, v.2, n.3, p.1-19. 2013.

SOUZA, J.A. et al. Biofotogrametria confiabilidade das medidas do protocolo do software para avaliação postural (SAPO). Revista Brasileira de Cineantropometria e Desempenho Humano, v. 13, n.4, p.299-305. 2011.

TILLEY, A.; DREYFUSS, $H$. The Measure of Man and Woman, Human factors design, Whitney Library of Design, New York, USA, 1993. Apud: BERGMANA, C., et al. Implementation of suitable comfort model for posture and motion prediction in DHM supported vehicle design. 6th International Conference on Applied Human Factors and Ergonomics (AHFE 2015). Procedia Manufacturing. Vol. 3, p. 3753 - 3758. 2015. p. 3755.

ZENK, R.; et al. Technical note: Spine loading in automotive seating. Applied Ergonomics. Vol. 43, n. 2, p. 290-295. 2012. 